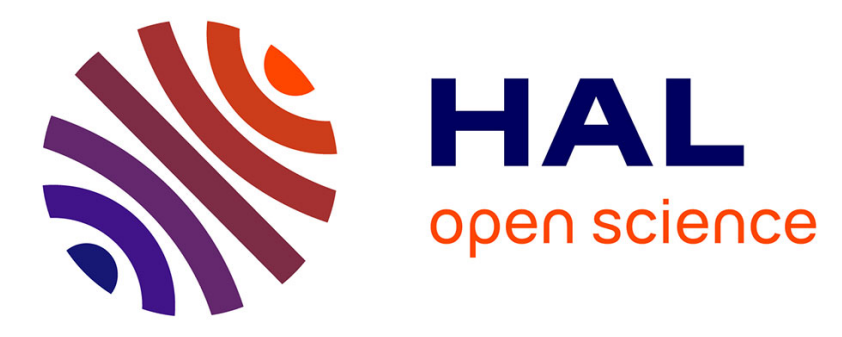

\title{
Perfusion imaging and clinical outcome in acute ischemic stroke with large core
}

Pierre Seners, Catherine Oppenheim, Guillaume Turc, Jean- François Albucher, Adrien Guenego, Nicolas Raposo, Soren Christensen, Lionel Calvière, Alain Viguier, Jean Darcourt, et al.

\section{To cite this version:}

Pierre Seners, Catherine Oppenheim, Guillaume Turc, Jean- François Albucher, Adrien Guenego, et al.. Perfusion imaging and clinical outcome in acute ischemic stroke with large core. Annals of Neurology, 2021, Online ahead of print. 10.1002/ana.26152 . inserm-03287979

\section{HAL Id: inserm-03287979 https://www.hal.inserm.fr/inserm-03287979}

Submitted on 16 Jul 2021

HAL is a multi-disciplinary open access archive for the deposit and dissemination of scientific research documents, whether they are published or not. The documents may come from teaching and research institutions in France or abroad, or from public or private research centers.
L'archive ouverte pluridisciplinaire HAL, est destinée au dépôt et à la diffusion de documents scientifiques de niveau recherche, publiés ou non, émanant des établissements d'enseignement et de recherche français ou étrangers, des laboratoires publics ou privés. 


\section{Research article}

\section{Perfusion imaging and clinical outcome in acute ischemic stroke with large core}

Pierre Seners, ${ }^{1,2}$ MD-PhD; Catherine Oppenheim*, ${ }^{3}$ MD-PhD; Guillaume Turc*, ${ }^{1}$ MD-PhD; JeanFrançois Albucher, ${ }^{4} \mathrm{MD}$; Adrien Guenego, ${ }^{5} \mathrm{MD}$; Nicolas Raposo, ${ }^{4} \mathrm{MD}-\mathrm{PhD}$; Soren Christensen, ${ }^{6}$ $\mathrm{PhD}$; Lionel Calvière, ${ }^{4} \mathrm{MD}$; Alain Viguier, ${ }^{4} \mathrm{MD}$; Jean Darcourt, ${ }^{5} \mathrm{MD}$; Anne-Christine Januel, ${ }^{5} \mathrm{MD}$; Michael Mlynash, ${ }^{6} \mathrm{MD}$; Agnes Sommet, ${ }^{7} \mathrm{MD}-\mathrm{PhD}$; Claire Thalamas, ${ }^{7} \mathrm{MD}$; Igor Sibon, ${ }^{8} \mathrm{MD}-\mathrm{PhD}$; Vanessa Rousseau, ${ }^{7} \mathrm{PhD}$; Thomas Tourdias, ${ }^{9} \mathrm{MD}-\mathrm{PhD}$; Patrice Menegon, ${ }^{9} \mathrm{MD}$; Fabrice Bonneville, ${ }^{5}$ MD-PhD; Mikael Mazighi, ${ }^{10}$ MD-PhD; Sylvain Charron, ${ }^{3} \mathrm{PhD}$; Laurence Legrand, ${ }^{3}$ MD-MSc; Christophe Cognard, ${ }^{5}$ MD-PhD; Gregory W. Albers, ${ }^{6}$ MD; Jean-Claude Baron, ${ }^{1}$ MD-ScD; Jean-Marc Olivot, ${ }^{4} \mathrm{MD}-\mathrm{PhD}$; on behalf of the FRAME investigators.

1: Neurology Department, GHU Paris psychiatrie et neurosciences, Institut de Psychiatrie et Neurosciences de Paris (IPNP), INSERM U1266, Université de Paris, FHU Neurovasc, Paris, France. 2 : Neurology Department, Hôpital Fondation A. de Rothschild, Paris, France.

3: Radiology Department, GHU Paris psychiatrie et neurosciences, Institut de Psychiatrie et Neurosciences de Paris (IPNP), INSERM U1266, Université de Paris, FHU Neurovasc, Paris, France.

4: Acute Stroke Unit, Hôpital Pierre-Paul Riquet, Centre Hospitalier Universitaire de Toulouse and Toulouse NeuroImaging Center, Université de Toulouse, Inserm, UPS, France

5 : Department of Neuroradiology, Centre Hospitalier Universitaire Toulouse, France.

6 : Stanford Stroke Center, Stanford University, CA, USA.

7 : Clinical Investigation Center, Centre Hospitalier Universitaire de Toulouse, France.

8: Unité Neurovasculaire, Centre Hospitalier Universitaire de Bordeaux, Université de Bordeaux, France.

9: Department of Neuroradiology, Centre Hospitalier Universitaire de Bordeaux, Université de Bordeaux, France

10 : Department of Interventional Neuroradiology, Hôpital Fondation A. de Rothschild, Paris, France.

The list of FRAME collaborators is presented in supplementals.

*Equal participation

Correspondence to Prof. Jean-Claude Baron, Neurology Department, Sainte-Anne Hospital; 1, rue Cabanis, 75014 Paris, France. E-mail: jean-claude.baron@inserm.fr

Phone: 331456586 34. Fax: 33145658794

Running head title: Perfusion imaging in acute stroke with large core

Number of words in abstract: 250

Number of words in the body of manuscript: 3464

Number of words in introduction: 460

Number of words in discussion: 1164

Number of tables: 4; Number of figures: 4. 


\section{Summary for social media if Published}

Twitter accounts: @PSeners

What is the current knowledge on the topic? (one to two sentences)

Mechanical thrombectomy is currently not recommended for acute stroke with large vessel occlusion and a large volume of irreversibly injured tissue ("core"). Whether some of these patients benefit from mechanical thrombectomy remains unsettled.

\section{What question did this study address?}

Could perfusion imaging identify a subset of patients with large core and large vessel occlusion who benefit from mechanical thrombectomy?

\section{What does this study add to our knowledge?}

Our study shows that roughly half of large core patients with large vessel occlusion admitted in an early time window have significant critically ischemic but salvageable tissue as evaluated by perfusion imaging, and suggests that, as compared to medical treatment alone, mechanical thrombectomy improves functional outcome in the latter subgroup only.

\section{How might this potentially impact on the practice of neurology?}

Perfusion imaging may help select which patients with large core and large vessel occlusion should be considered for mechanical thrombectomy. 


\section{Abstract}

Objective: Mechanical thrombectomy (MT) is not recommended for acute stroke with large vessel occlusion (LVO) and a large volume of irreversibly injured tissue (“core"). Perfusion imaging may identify a subset of patients with large core who benefit from MT.

Methods: We compared two cohorts of LVO-related patients with large core ( $>50 \mathrm{ml}$ on diffusionweighted-imaging or CT-perfusion using RAPID), available perfusion imaging, and treated within 6 hrs from onset by either MT+Best Medical Management (BMM) in one prospective study, or BMM alone in the pre-MT era from a prospective registry. Primary outcome was 90-day modified Rankin Scale $\leq 2$. We searched for an interaction between treatment group and amount of penumbra as estimated by the mismatch ratio (MMRatio=critical hypoperfusion/core volume).

Results: Overall, 107 patients were included (56 MT+BMM + $51 \mathrm{BMM})$ : Mean age was $68 \pm 15 \mathrm{yrs}$, median core volume 99ml (IQR: 72-131) and MMRatio 1.4 (IQR: 1.0-1.9). Baseline clinical and radiological variables were similar between the 2 groups, except for a higher intravenous thrombolysis rate in the BMM group. The MMRatio strongly modified the clinical outcome following MT ( $P_{\text {interaction }}<0.001$ for continuous MMRatio); MT was associated with a higher rate of good outcome in patients with, but not in those without, MMRatio $>1.2$ (adjusted OR $[95 \% \mathrm{CI}]=6.8[1.7-27.0]$ vs. 0.7 [0.1-6.2], respectively). Similar findings were present for MMRatio $\geq 1.8$ in the subgroup with core $\geq 70 \mathrm{ml}$. Parenchymal hemorrhage on follow-up imaging was more frequent in the MT+BMM group regardless of the MMRatio.

Interpretation: Perfusion imaging may help select which patients with large core should be considered for MT. Randomized studies are warranted. 


\section{Introduction}

Mechanical thrombectomy (MT) is currently recommended over best medical management (BMM; as per the attending physician) alone in acute ischemic stroke (AIS) patients with large vessel occlusion (LVO) and moderate-to-severe clinical deficits but without large irreversibly injured tissue ("core") who can be treated in the early time window. ${ }^{1,2}$ Because LVO-related AIS patients with large core are expected to have high rates of poor functional outcomes regardless of treatment, ${ }^{3,4}$ they were largely excluded from the pivotal MT trials, and current guidelines do not recommend MT in this population. ${ }^{1,}$

${ }^{2}$ Nonetheless, subgroup analysis of the MT trials has suggested that MT may improve functional outcome -as compared to BMM alone- in this population, ${ }^{4}$ consistent with several observational studies. ${ }^{5-10}$ However, uncertainty remains about which patients with large core may benefit from MT.

Although one major candidate for identifying responders to MT among large core LVO patients is quantitative perfusion imaging, available data is limited because perfusion imaging, when performed, was typically used to exclude patients with absent "penumbra", i.e., critically ischemic but salvageable tissue. ${ }^{4-6}$ Hence, although the subgroup analysis focusing on patients with large core from the pivotal MT trials showed greater functional improvement in MT+BMM vs. BMM alone, the vast majority of the included patients had substantial penumbra, namely $99 \%$ and $95 \%$ as estimated by a hypoperfusion/core ratio (mismatch ratio, MMRatio) $>1.2$ and $\geq 1.8$, respectively. ${ }^{4}$ This also applies to an observational study that focused on patients with persistent penumbra. ${ }^{5}$ Another observational study found increasing benefit of MT+BMM as compared to BMM alone with larger penumbra, with MT not beneficial with MMRatio $<1.8,{ }^{6}$ yet again the results of penumbral imaging likely influenced decision-making as suggested by the mean MMRatio of 1.8 in the MT group. Overall, therefore, even if available data suggest that MT may be beneficial in large-core AIS with persistent penumbra, whether MT also benefits patients without persistent penumbra is unknown. FRAME (FRench Acute multimodal imaging study to select Patients for MEchanical Thrombectomy), a prospective cohort of patients treated by MT within 6hrs after onset where treatment decision was made blinded to the results of baseline perfusion imaging, revealed two important findings ${ }^{11}$ : first, only half of the patients with a large core $(>70 \mathrm{~mL})$ had penumbra defined by a MMRatio $>1.2$; and second, only this subgroup derived benefit from MT-induced reperfusion, as compared to no-reperfusion. ${ }^{11}$ 
We therefore hypothesize that large core patients with substantial persistent penumbra will benefit from MT, but not those with imaging suggestive of completed infarction. To test this hypothesis, we compared large core LVO patients treated with MT+BMM in the FRAME study to similar patients treated with BMM alone in the pre-MT era as a function of baseline penumbra status, and specifically assessed the interaction between presence of penumbra on functional outcome in MT+BMM $v s$. BMM alone. 


\section{Methods}

\section{Study design and data sources}

This observational retrospective comparative study included consecutive patients from two prospectively collected databases that fulfilled the following criteria: (1) AIS admitted within 6hrs from last seen well with LVO on baseline imaging (internal carotid artery [ICA], first [M1] or second [M2] segment of middle cerebral artery); (2) absence of pre-stroke significant disability (modified Rankin Scale score $[\mathrm{mRS}]<2$ ); (3) perfusion imaging performed before treatment decision; and (4) infarct core volume $>50 \mathrm{ml}$ on either Diffusion-Weighted Imaging (DWI) or CT-perfusion. ${ }^{5,7,12}$

\section{$M T+B M M$ group}

All patients included in the FRAME study who fulfilled the above criteria were extracted. The detailed methodology and main results of FRAME have been previously published. ${ }^{11}$ Briefly, FRAME was a prospective cohort study that aimed to test whether the absence of target mismatch was predictive of poor response to reperfusion. To this aim, 218 LVO-related AIS patients treated by MT+BMM within 6 hours from last seen well were enrolled from January 2017 to February 2019 in two French comprehensive stroke centers (Toulouse and Bordeaux University Hospital) using mostly MRI as routine first-line diagnostic tool in candidates for reperfusion therapy. Perfusion imaging (either perfusion-weighted imaging [PWI] on MRI or CT-perfusion) was performed in all included patients but both treatment decision and 3-month mRS evaluation were performed blinded to the results of baseline perfusion imaging: both centers contracted with iSchema View to install a specific version of the RAPID software that did not process the DWI/PWI/CT-perfusion maps on-site and automatically sent them to a secure server. These maps were not accessible on any on-site clinical imaging system (emails, Picture Archiving and Communication System, etc), and treatment decisions and patient management were therefore made using standard MRI sequences only, namely DWI/gradient echo/fluid attenuated inversion recovery and MR-angiography, or noncontrast CT and CTangiography. The DWI/PWI and CT-perfusion maps were processed a posteriori, after recruitment completion. Importantly, a large infarct core as assessed by a low Alberta Stroke Program Early CT score was not an exclusion criterion and decision to carry out MT was based on clinical judgment. 
The trial protocol was approved by the French Ethical Committee (Comité de Protection des Personnes Sud-Ouest et Outre Mer III) on October 5, 2016, and was authorized by the French Health Authority. Every patient or his/her legal representative signed a written informed consent at inclusion.

\section{$B M M$ only group}

All consecutive patients who fulfilled the above criteria and received BMM alone from January 2006 to December 2014 were extracted from the Sainte-Anne Hospital prospective registry, a time period where MT was rarely performed, except in the setting of trials. In this center, MRI was systematically implemented as routine first-line imaging tool in candidates for reperfusion therapy. The MRI admission protocol included DWI, fluid-attenuated inversion recovery, gradient echo imaging, intracranial MR-angiography, and PWI whenever feasible with no delay. Patients in whom MRI was contraindicated underwent CT and CT-angiography; CT-perfusion was performed whenever feasible with no delay. During this time period, the post-processing of thresholded DWI and perfusion maps was not automated and required a time-consuming manual post-processing using a local software. Therefore these maps were typically not used for decision-making. Importantly, a large baseline DWI lesion was not considered a contra-indication for IVT. ${ }^{13}$ Part of this cohort has been reported in previous publications. ${ }^{6,13}$

In accordance with the French legislation, each patient was informed of his/her participation in this study and was offered the possibility to withdraw. However, as this study only implied retrospective analysis of anonymized data collected as part of routine care, formal approval by an ethics committee was not required.

\section{Clinical and radiological data}

The following variables were collected for both groups: age, gender, vascular risk factors, pre-stroke anti-thrombotic medication, time between symptom onset and qualifying imaging, blood pressure on admission, intravenous thrombolysis administration, NIHSS score on admission and at $24 \mathrm{~h}$ follow-up, and 3-month mRS score. For the MT+BMM group, we additionally collected delay between (1) symptoms onset and groin puncture and (2) symptoms onset and end of procedure. All included patients underwent either MRI with PWI, or CT, CT-angiography and CT-perfusion on admission at 
the comprehensive stroke center, and follow-up MRI or CT within $\sim 24$ hrs following admission. A central imaging reading was performed separately in each treatment group, blinded from clinical outcomes. The following variables were collected: (1) infarct core (defined by MRI as an apparent diffusion coefficient less than $620 \mu \mathrm{m}^{2} / \mathrm{s}$ or CT-perfusion as relative cerebral blood flow less than $30 \%$ of normal brain blood flow) and time-to-maximum (Tmax) $>6$ s volumes, processed by the RAPID software using DWI/PWI or CT-perfusion; (2) mismatch ratio (MMRatio), defined $\operatorname{Tmax}>6 \mathrm{sec}$ volume/core volume; (3) occlusion site, divided into the following categories: ICA (intracranial T/L occlusions or tandem cervical ICA + M1 or M2), M1 or M2; and (4) intracranial haemorrhage on follow-up imaging, based on the European Cooperation Acute Stroke Study. ${ }^{14}$ For the MT+BMM group, successful reperfusion was defined as the modified Thrombolysis in Cerebral Infarction (mTICI) score of $2 \mathrm{~b}$ or 3.

\section{Clinical outcomes}

The primary outcome was good functional outcome, defined as 90-day mRS 0-2. Shift analysis of the mRS and mRS 0-3 were used as secondary outcomes. Safety outcome was parenchymal haemorrhage 1 or 2 on $\sim 24$-hr follow-up imaging according to the European Cooperative Acute Stroke Study. ${ }^{14}$

\section{Statistical analysis}

Categorical variables were described as numbers and percentages and continuous variables as mean \pm standard deviation or median (interquartile range), as appropriate. The two treatment groups were compared for baseline clinical and radiological variables using Student $t$ test or Mann-Whitney $U$ test for continuous variables and Chi-square or Fisher's exact test for categorical variables, as appropriate. The association between treatment group and each outcome was estimated through odds ratios (ORs) and their 95\% confidence intervals (95\% CIs), calculated in univariable and multivariable logistic regression. Binary logistic regression models were used, except for the modelization of better functional outcome across the whole range of the mRS, which was based on ordinal logistic regression. We a priori decided to adjust each model on i) baseline variables showing significant difference between the two treatment groups; and ii) infarct core volume, which is a strong predictor

of 3-month outcome. ${ }^{3,4}$ Potential heterogeneity in treatment effect depending on MMRatio, either 
dichotomized (using 1.2 or 1.8 as cut-offs, i.e., the two main cut-offs used in clinical trials ${ }^{12,15,16}$ ) or as a continuous variable, was assessed in logistic models with calculation of $P$ values for interaction $\left(P_{\text {interaction }}\right)$. To further decipher the role of core volume on the heterogeneity in treatment effect size as a function of MMRatio we made 1) graphical representations of the probability of mRS 0-2 according to MMRatio and treatment group as a function of increasing core volumes; and 2) a sensitivity analysis for patients with core volume $\geq 70 \mathrm{ml}$, with $\mathrm{mRS}$ 0-3 as clinical outcome. ${ }^{6}$ Statistical analyses were performed using SAS 9.4 (SAS Institute, Cary, NC). Two-tailed $P<0.05$ was considered significant. 


\section{Results}

\section{Study population}

One hundred and seven patients were included (56 with MT+BMM; and 51 with BMM alone). Mean age was $68 \pm 15$ years, 41 (38\%) patients were female, 105 (98\%) patients had MRI and median (IQR) core volume and MMRatio were 99ml (72-131) and 1.4 (1.0-1.9), respectively. The proportion of included patients without mismatch was 43\% (46/107) and 64\% (69/107) using the 1.2 and 1.8 MMRatio cut-offs, respectively. Table 1 summarizes baseline characteristics in the 2 groups. The 2 groups were well balanced for all variables -including core volume and MMRatio- except for IVT rate, which was higher in the BMM alone group and was therefore adjusted for in subsequent analysis.

\section{Association between treatment group and efficacy outcomes according to MMRatio}

Primary outcome (mRS 0-2)

The unadjusted and adjusted relationships between treatment group and good outcome according to dichotomized MMRatio are presented in Table 2. Using both the 1.2 and 1.8 MMRatio cut-offs, there was a significant treatment group*MMRatio interaction, with $\mathrm{MT}+\mathrm{BMM}$ associated with better clinical outcomes in patients with a favorable MMRatio, but not without $\left(P_{\text {interaction }}=0.036\right.$ and $<0.001$ for MMRatio 1.2 and 1.8, respectively). The predicted probability of $\mathrm{mRS} 0-2$ as a function of continuous MMRatio and treatment group is presented in Figure 1A, which shows that the greater the MMRatio, the higher the probability of good outcome following MT+BMM but the lower the probability of good outcome following BMM alone $\left(P_{\text {interaction }}<0.001\right)$.

To further decipher associations between core volume and MMRatio on one hand, and functional outcome on the other hand, Figure 2 shows the predicted probability of good outcome as a function of continuous MMRatio, treatment group and increasing core volume (from 50 to 200ml), whereas Figure 3 shows the predicted probability of good outcome according to core volume (expressed as a continuous variable) and MMRatio in the MT+BMM group. These figures show that increasing core volume mitigates the relationship between the MMRatio and the clinical effect of $\mathrm{MT}+\mathrm{BMM} v s$. BMM alone. 
Secondary outcomes ( $m R S$ 0-3 and shift analysis of the $m R S$ )

The relationships between treatment group and mRS 0-3 according to dichotomized or continuous MMRatio are presented in Table 2 and Figure 1B, respectively, showing very similar results as with mRS $0-2\left(P_{\text {interaction }}=0.11\right.$ and $<0.001$ for dichotomized MMRatio with the 1.2 and 1.8 cut-offs, respectively, and $P_{\text {interaction }}=0.005$ for continuous MMRatio). Also, as shown on Figure 4, there was a treatment group*MMRatio interaction in the shift analysis of the mRS scores for the 1.8 cut-off $\left(P_{\text {interaction }}=0.008\right)$, and a non-significant trend for the 1.2 cut-off $\left(P_{\text {interaction }}=0.12\right)$. MT was associated with a favorable shift in the overall distribution of 90-day mRS scores for patients with significant MMRatio (MMRatio $>1.2$ : common $\mathrm{OR}=2.9$ [1.1-7.5], $P=0.03$; MMRatio $\geq 1.8$ : common $\mathrm{OR}=6.3$ [1.7-22.7], $P=0.005$ ), but not without $(M M R a t i o \leq 1.2$ : common $\mathrm{OR}=1.1 \quad[0.4-3.0], \quad P=0.93$; MMRatio $<1.8$ : common $\mathrm{OR}=1.0[0.4-2.2], P=0.91)$.

\section{Association between treatment group and safety outcome according to MMRatio}

The unadjusted and adjusted relationships between treatment group and safety outcome (parenchymal hemorrhage) according to dichotomized MMRatio are presented in Table 3. Using both the 1.2 and 1.8 MMRatio cutoffs, there was no treatment group*MMRatio interaction $\left(P_{\text {interaction }}=0.90\right.$ and 0.64 for MMRatio 1.2 and 1.8, respectively), with $\mathrm{BMM}+\mathrm{MT}$ being associated with higher odds of parenchymal hemorrhage in both patients with and without significant MMRatio.

\section{Sensitivity analysis for core $\geq 70 \mathrm{ml}$}

Eighty-three patients had baseline core $\geq 70 \mathrm{ml}$ ( 37 and 46 in the BMM and MT+BMM groups, respectively). The unadjusted and adjusted relationships between treatment group and mRS 0-3 according to dichotomized MMRatio are presented in Table 4. In this subgroup, there was a significant treatment group*MMRatio interaction regarding mRS 0-3 when considering the higher MMRatio cutoff only $\left(\right.$ MMRatio $\leq 1.2: \mathrm{OR}=1.4[0.4-5.0]$, MMRatio $>1.2: \mathrm{OR}=2.2[0.6-8.0], P_{\text {interaction }}=$ 0.66; MMRatio<1.8: OR=1.1 [0.4-2.9], MMRatio $\left.\geq 1.8: \mathrm{OR}=17.8[0.7-437.4], P_{\text {interaction }}=0.03\right)$. 


\section{Discussion}

This novel study aimed to compare the rates of favorable clinical outcomes as well as safety outcomes of MT+BMM versus BMM alone in patients with LVO-related AIS and core volume $>50 \mathrm{ml}$ who presented $\leq 6 \mathrm{hrs}$, as a function of the amount of perfusion mismatch present on baseline imaging. Three salient findings emerged. First, a high proportion of the included patients across the two groups had no mismatch, namely $43 \%$ and $64 \%$ using the 1.2 and 1.8 MMRatio cut-offs, respectively. Second, consistent with our hypothesis, MT $+\mathrm{BMM}$ was associated with a significantly higher rate of good functional outcome over BMM alone in the subgroup with mismatch, but not in the subgroup without. Third, MT+BMM was associated with higher odds of parenchymal hemorrhage regardless of the presence or extent of mismatch, yet this did not outweight the benefits from MT in patients with mismatch. These three points will be discussed in succession.

The prevalence of mismatch in both cohorts was far lower than previously reported, ${ }^{4-7}$ with roughly 1 in 2 patients with baseline core $>50 \mathrm{ml}$ showing no remaining salvageable tissue. This is most likely because in both cohorts a large core was not an a priori exclusion criterion for reperfusion therapy, thereby affording an assessment of the true prevalence of mismatch in this population. This in turn calls into question the previously mentioned studies suggesting benefit from MT in large-core populations where perfusion imaging, when available, was, or likely was, used in decision-making. ${ }^{4-6}$

Second, the probability of good functional outcome increased in proportion with the MMRatio in the MT+BMM group, whereas it conversely decreased in the BMM group (Figure 1). The benefit from $\mathrm{MT}+\mathrm{BMM}$ relative to $\mathrm{BMM}$ alone therefore increased in proportion with increasing MMratio. Further inspection of our data shows that this relationship was influenced by baseline core volume. This may be explained by both the expected decreasing extent of mismatch and increasing rates of post-MT poor clinical outcome with increasing core volumes (Figures 2 and 3). ${ }^{3,4}$ Importantly MT+BMM was not associated with improved functional outcomes over BMM alone in patients with no mismatch, showing even a trend for worse outcomes, in line with 3 previous reports. ${ }^{4,}$, 11 First, among MTtreated large core LVO patients in FRAME, only those with a mismatch appeared to benefit from MTinduced reperfusion. ${ }^{11}$ Second, among the only 34 patients with perfusion imaging having no 
mismatch (using the 1.8 MMRatio cut-off) from the pivotal MT trials, ${ }^{4} \mathrm{MT}+\mathrm{BMM}$ did not improve functional outcome (common $\mathrm{OR}=0.87$ [95\%CI 0.20-3.81], $P=0.85$ ). Last, an observational study in large core LVO patients showed no benefit from MT in case of MMRatio <1.8. ${ }^{6}$ However, the unusually high prevalence of large mismatch in the MT group in the latter study ${ }^{6}$ suggests that, contrary to FRAME, perfusion imaging may have influenced indication for MT, thereby hindering interpretation of findings in the no-mismatch situation.

We also found that MT+BMM was associated with higher rates of parenchymal hemorrhage, both in patients with and without mismatch. This finding is consistent with previous studies reporting higher rates of any intracranial hemorrhage ${ }^{7}$ or symptomatic intracranial hemorrhage ${ }^{17}$ in large core patients treated by MT. Reperfusion of a large amount of core -a much more frequent occurrence following MT+BMM than BMM alone- may enhance the odds of intracranial hemorrhage, as previously documented following IVT. ${ }^{18}$ Interestingly, the occurrence of even mild, 'asymptomatic' hemorrhagic transformation appears associated with worse outcome after successful MT. ${ }^{19,}{ }^{20}$ In patients without mismatch, the lack of clinical benefit from MT on functional outcome, together with higher odds of parenchymal hemorrhage, suggests that MT might be not just "not beneficial", but also potentially harmful to these patients, as supported by the trends seen here. Conversely, the major benefits from penumbral salvage following MT found in patients with significant mismatch clearly outweighed any negative impact of intracranial hemorrhage. Importantly, the higher rate of parenchymal hemorrhage found in the MT+BMM group cannot be attributed to IVT, since the rate of IVT was higher in the BMM alone group (90\% vs 57\%).

Documenting a high prevalence of no mismatch, together with a strong influence of MMRatio on clinical outcome, sheds new light on current debates regarding the best treatment strategy for acute stroke with LVO and large core. Because large core was an exclusion criterion in most of the pivotal MT trials, the benefit of MT+BMM over BMM alone has remained uncertain, and several ongoing randomized trials are comparing $\mathrm{MT}+\mathrm{BMM} v s$. $\mathrm{BMM}$ alone in patients with large core as defined by an Alberta Stroke Program Early CT score $\leq 5$ (NCT03811769 and NCT03094715). Our results suggest that this design will inevitably result in merging patients with distinct underlying perfusion 
profiles predicting distinct responses to MT, namely, roughly half of the patients will have a mismatch, and half will not. Consequently, a positive result from these trials would extend the indication for MT to the latter population, in which the benefit/risk profile of MT appears unfavorable, while a negative result would exclude the former population from a treatment that likely is beneficial. In the current emerging era of personalized medicine, our data suggest that future trials in large core LVO populations may consider adding mismatch as a selection criterion in order to enhance their chance of positive and meaningful findings. Finally, given the huge benefits from MT in LVO-related AIS patients with small core but mismatch up to $24 \mathrm{hrs}$ after stroke onset, ${ }^{16,21}$ it may be speculated that MT could also be beneficial in patients with large core and mismatch in such late time-windows. This warrants further studies.

\section{Study limitations}

First, this study has limitations inherent to its observational design. Notably, although there were only few between-group differences in baseline characteristics, unmeasured confounding factors affecting both treatment decision and functional outcome may have been overlooked. In addition, our samples are relatively small, thereby limiting statistical power. Second, because the two treatment groups were enrolled at distinct time periods, we cannot exclude the existence of unmeasured confounding factors that may have changed over time and impacted outcomes. Third, our findings reflect clinical practice in only three institutions, and generalizability is therefore uncertain. Replication in an independent dataset is therefore warranted. Fourth, based on previous work, ${ }^{5,72}$ we opted for $>50 \mathrm{ml}$ as large core definition, whereas others advocate the $70 \mathrm{ml}$ cut-off. ${ }^{4,6,13,15}$ However, the sensitivity analysis using $70 \mathrm{ml}$ and the mRS 0-3 outcome -albeit limited by the smaller sample- showed similar trends. Last, the vast majority of the patients were evaluated with MRI, which reduces the generalizability of our results since $\mathrm{CT}$ is the first-line imaging modality in most centers worldwide. Although direct comparisons of MR vs. CT-based perfusion datasets has shown that both techniques can estimate hypoperfused tissue with comparable accuracy ${ }^{22}$ and previous trials using either modality for patient selection did not report significant modality-related outcome differences ${ }^{16}{ }^{1}$ ischemic core assessment on CT-perfusion in the very early time window may be less accurate, ${ }^{23-26}$ and differences in CT- 
perfusion processing software add further variability. ${ }^{27}$ Therefore, further studies using CT-perfusion are warranted before translating the present findings into clinical practice.

\section{Conclusions}

Our findings document that among large-core LVO stroke populations assessed $\leq 6 \mathrm{hrs}$ from last-seenwell, roughly half have mismatch, and only this subgroup appears to benefit from MT. In addition, MT+BMM was associated with increased rates of parenchymal hemorrhage over BMM alone regardless of the baseline perfusion imaging profile, calling into question the rationale for MT in large core LVO patients without mismatch. Finally, this study provides evidence that advanced imaging may be worth considering for patient selection in future trials testing MT in LVO-related large-core AIS. 
Acknowledgement: FRAME (French Acute Multimodal Imaging Study to Select Patients for Mechanical Thrombectomy) was supported by a research grant from the French Ministry of Health, Clinical Research Hospital Program 2015 (PHRCI-15-076). The study sponsor, CHU de Toulouse, has no role in study design, collection, analysis, and interpretation of data; writing of this manuscript; or the decision to submit for publication.

\section{Authors contributions:}

PS, JCB and JMO conceived and designed the study.

PS, CO, GT, JFA, AG, NR, SChr, LC, AV, JD, ACJ, MMl, AS, CT, IS, VR, TT, PM, FB, MMa, SCha, LL, CC, GWA, JCB and JMO acquired and analyzed the data.

PS, JCB and JMO drafted the manuscript.

FRAME collaborators names and affiliations: see supplementary file.

\section{Potential conflict of interests:}

Gregory Albers, consulting for Genentech, and ownership interest and consultant for iSchemaView. Soren Christensen, Consulting, Ischema View.

The other authors report no conflicts. 
Table 1. Baseline characteristics of the 2 treatment groups

\begin{tabular}{|c|c|c|c|}
\hline & $\begin{array}{c}\text { MT+BMM } \\
n=56\end{array}$ & $\begin{array}{c}\text { BMM } \\
\mathrm{n}=51\end{array}$ & $\boldsymbol{P}$ \\
\hline Age (years) & $73(61-80)$ & $69(57-80)$ & 0.44 \\
\hline Male gender & $32(57)$ & $34(67)$ & 0.31 \\
\hline \multicolumn{4}{|l|}{ Patient history } \\
\hline Hypertension & $32(57)$ & $28(55)$ & 0.82 \\
\hline Diabetes mellitus & $10(18)$ & $8(16)$ & 0.76 \\
\hline Statins & $12(21)$ & $17(33)$ & 0.17 \\
\hline Current smoking & $11(20)$ & $8(16)$ & 0.59 \\
\hline Atrial fibrillation & $9(16)$ & $8(16)$ & 0.96 \\
\hline Antiplatelets & $13(23)$ & $15(29)$ & 0.47 \\
\hline Anticoagulation & $5(9)$ & $0(0)$ & 0.06 \\
\hline \multicolumn{4}{|l|}{ Baseline clinical characteristics } \\
\hline NIHSS score & $20(16-25)$ & $19(16-23)$ & 0.26 \\
\hline Systolic blood pressure $(\mathrm{mmHg})^{\mathrm{a}}$ & $150(135-168)$ & $159(141-175)$ & 0.13 \\
\hline Diastolic blood pressure $(\mathrm{mmHg})^{\mathrm{a}}$ & $80(70-90)$ & $80(70-92)$ & 0.61 \\
\hline \multicolumn{4}{|l|}{ Baseline imaging } \\
\hline Onset-to-imaging time (min) & $145(108-223)$ & $126(95-174)$ & 0.08 \\
\hline MRI & $55(98)$ & $50(98)$ & 1.0 \\
\hline Right side & $30(54)$ & $22(43)$ & 0.28 \\
\hline Occlusion site & & & 0.49 \\
\hline ICA & $19(34)$ & $12(24)$ & \\
\hline M1 & $28(50)$ & $29(57)$ & \\
\hline M2 & $9(16)$ & $10(20)$ & \\
\hline Core volume, $\mathrm{ml}$ & $105(76-133)$ & $97(65-124)$ & 0.16 \\
\hline$\geq 70 \mathrm{ml}$ & $46(82)$ & $37(73)$ & 0.24 \\
\hline Tmax $>6$ sec volume, $\mathrm{ml}$ & $153(117-189)$ & $139(100-183)$ & 0.16 \\
\hline Mismatch volume, $\mathrm{ml}$ & $46 \pm 59$ & $40 \pm 57$ & 0.60 \\
\hline Mismatch ratio & $1.5(1.1-1.9)$ & $1.3(0.9-1.9)$ & 0.49 \\
\hline$>1.2$ & $33(59)$ & $28(55)$ & 0.67 \\
\hline$\geq 1.8$ & $20(36)$ & $18(35)$ & 0.96 \\
\hline \multicolumn{4}{|l|}{ Treatment following imaging } \\
\hline IV-thrombolysis & $32(57)$ & $46(90)$ & $<0.01$ \\
\hline Onset-to-IVT time, $\min$ & $152(129-188)$ & $170(135-200)$ & 0.11 \\
\hline Onset-to-puncture time, $\min$ & $215(179-295)$ & NA & NA \\
\hline Onset-to-end of procedure, min & $261(214-354)$ & NA & NA \\
\hline mTICI 2b-3 & $46(82)$ & NA & NA \\
\hline
\end{tabular}

a: 9 missing values in the MT+BMM group.

Abbreviations: BMM indicates best medical management; ICA, internal carotid artery; IVT, intravenous thrombolysis; M1; first segment of middle cerebral artery; M2, second segment of middle cerebral artery; MRI, magnetic resonance imaging; MT, mechanical thrombectomy; mTICI, modified Thromblysis in Cerebral 
Infarction scale; NA, not appropriate; NIHSS, National Institutes of Health Stroke Scale; Tmax, time-tomaximum.

Continuous variables are presented as mean \pm standard deviation or median (interquartile range), and categorical variables as number (percentages). 
Table 2. Relationships between treatment group and primary (mRS 0-2) and secondary (mRS 0-3) outcomes according to dichotomized mismatch ratio

\begin{tabular}{|c|c|c|c|c|c|c|}
\hline & & $\begin{array}{c}\text { MT+BMM } \\
\mathrm{N}=56\end{array}$ & $\begin{array}{l}\text { BMM } \\
\mathrm{N}=51\end{array}$ & $\begin{array}{c}\text { Unadjusted } \\
\text { analysis } \\
\text { OR (95\% CI) }\end{array}$ & $\begin{array}{c}\text { Adjusted } \\
\text { analysis } \\
\text { OR }(95 \% \mathrm{CI})\end{array}$ & $\begin{array}{c}P \text { for } \\
\text { interaction }\end{array}$ \\
\hline & MMRatio $\leq 1.2$ & $4 / 23(17)$ & $6 / 23(26)$ & $0.6(0.1-2.5)$ & $0.7(0.1-6.2)^{\mathrm{a}}$ & 0026 \\
\hline & MMRatio $>1.2$ & $16 / 33(49)$ & $5 / 28(18)$ & $4.3(1.3-14.1)$ & $6.8(1.7-27.0)^{\mathrm{a}}$ & 0.050 \\
\hline & MMRatio $<1.8$ & $8 / 36(22)$ & $10 / 33(30)$ & $0.7(0.2-1.9)$ & $1.2(0.3-4.7)^{\mathrm{a}}$ & \\
\hline & MMRatio $\geq 1.8$ & $12 / 20(60)$ & $1 / 18(6)$ & $17.2(2.5-118.4)^{b}$ & $20.9(2.8-157.4)^{\mathrm{a}, \mathrm{b}}$ & 0.001 \\
\hline & & & & & & \\
\hline & MMRatio $\leq 1.2$ & $11 / 23(48)$ & $11 / 23(48)$ & $1.0(0.3-3.2)$ & $2.2(0.50-10.1)^{\mathrm{a}}$ & 0 \\
\hline & MMRatio $>1.2$ & $18 / 33(55)$ & $7 / 28(25)$ & $3.6(1.2-10.8)$ & $4.9(1.5-16.0)^{\mathrm{a}}$ & 0.11 \\
\hline 常 & MMRatio $<1.8$ & $17 / 36(47)$ & $17 / 33(52)$ & $0.8(0.3-2.2)$ & $1.4(0.5-4.3)^{\mathrm{a}}$ & \\
\hline & MMRatio $\geq 1.8$ & $12 / 20(60)$ & $1 / 18(6)$ & $17.2(2.5-118.4)$ & $20.9(2.8-157.4)^{a, b}$ & .001 \\
\hline
\end{tabular}

a: Adjusted on core volume and treatment with intravenous thrombolysis (logistic regression) b: Firth correction Abbreviations: BMM indicates best medical management; MMRatio, mismatch ration; MT, mechanical thrombectomy; OR, odds ratio. 
Table 3. Relationships between treatment group and safety outcome (parenchymal hemorrhage) according to dichotomized mismatch ratio.

\begin{tabular}{|l|c|c|c|c|c|}
\hline & $\begin{array}{c}\text { MT+BMM } \\
\text { N=56 }\end{array}$ & $\begin{array}{c}\text { BMM } \\
\mathbf{N = 5 0}\end{array}$ & $\begin{array}{c}\text { Unadjusted analysis } \\
\text { OR (95\% CI) }\end{array}$ & $\begin{array}{c}\text { Adjusted analysis } \\
\text { OR (95\% CI) }\end{array}$ & $\begin{array}{c}\text { P for } \\
\text { interaction }\end{array}$ \\
\hline MMRatio $\leq \mathbf{1 . 2}$ & $9 / 23(39)$ & $2 / 23(9)$ & $6.8(1.3-36.0)$ & $4.5(0.7-28.7)^{\mathrm{b}}$ & \multirow{2}{*}{0.90} \\
\hline MMRatio $>\mathbf{1 . 2}$ & $14 / 33(42)$ & $3 / 27(11)$ & $5.9(1.5-23.5)$ & $4.8(1.2-18.8)^{\mathrm{b}}$ & \\
\hline \multicolumn{7}{|l|}{} & $5.9(1.2-27.7)^{\mathrm{b}}$ & \multirow{2}{*}{0.64} \\
\hline MMRatio <1.8 & $13 / 36(36)$ & $2 / 32(6)$ & $8.5(1.7-41.4)$ & $4.0(0.8-18.6)^{\mathrm{b}}$ & \\
\hline
\end{tabular}

a: 1 patient in the BMM group had no follow-up imaging because of early death, and was therefore excluded from this analysis.

b: Adjusted on core volume and treatment with intravenous thrombolysis (logistic regression); Firth correction.

Abbreviations: BMM indicates best medical management; MMRatio, mismatch ratio; MT, mechanical thrombectomy; OR, odds ratio. 
Table 4. Relationships between treatment group and mRS 0-3 according to dichotomized mismatch ratio, in the subgroup of patients with baseline ischemic core $\geq 70 \mathrm{ml}$.

\begin{tabular}{|c|c|c|c|c|}
\hline & $\begin{array}{c}\text { MT+BMM } \\
\text { N=46 }\end{array}$ & $\begin{array}{l}\text { BMM } \\
\mathbf{N}=\mathbf{3 7}\end{array}$ & $\begin{array}{c}\text { Unadjusted analysis } \\
\text { OR }(95 \% \text { CI })\end{array}$ & $P$ for interaction \\
\hline MMRatio $\leq 1.2$ & $11 / 23(48)$ & $7 / 18(39)$ & $1.4(0.4-5.0)$ & \multirow{2}{*}{0.66} \\
\hline MMRatio >1.2 & $10 / 23(44)$ & $5 / 19(26)$ & $2.2(0.6-8.0)$ & \\
\hline MMRatio < 1.8 & $16 / 35(46)$ & $12 / 27(44)$ & $1.1(0.4-2.9)$ & \multirow{2}{*}{$0.03^{b}$} \\
\hline MMRatio $\geq 1.8$ & $5 / 11(45)$ & $0 / 10(0)$ & $17.8(0.7-437.4)^{\mathrm{a}}$ & \\
\hline
\end{tabular}

a: Firth correction

b: Breslow-day test

Abbreviations: BMM indicates best medical management; MMRatio, mismatch ratio; MT, mechanical thrombectomy; OR, odds ratio. 


\section{Figure legends}

Figure 1. Predicted probability of primary (mRS 0-2, panel A) and secondary (mRS 0-3, panel B) outcomes according to mismatch ratio and treatment group (MT+BMM or BMM alone).
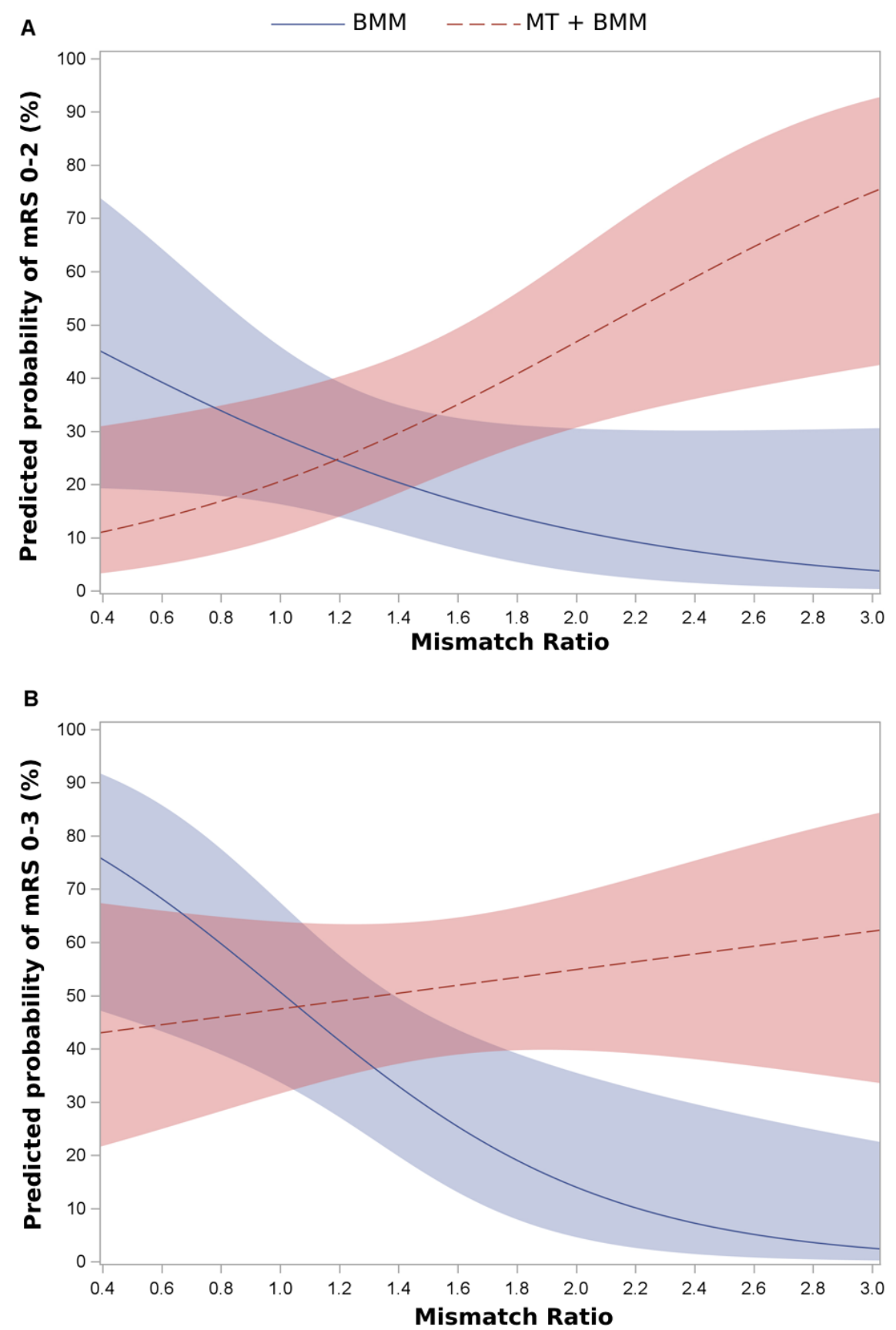

The regression curves are estimates of the probability of mRS 0-2 (A) or mRS 0-3 (B) according to the mismatch ratio. The red curve corresponds to $\mathrm{MT}+\mathrm{BMM}$ patients, and the blue curve to BMM alone patients. The shaded area corresponds to the $95 \%$ confidence interval (logistic regression model). There is a significant treatment group*mismatch ratio interaction for mRS 0-2 $\left(P_{\text {interaction }}<0.001\right)$ and mRS 0-3 $\left(P_{\text {interaction }}=0.005\right)$ probabilities.

Abbreviations: BMM indicates best medical management; MT, mechanical thrombectomy; mRS, modified Rankin scale. 
Figure 2. Probability of mRS 0-2 according to mismatch ratio, treatment group, and core volume.

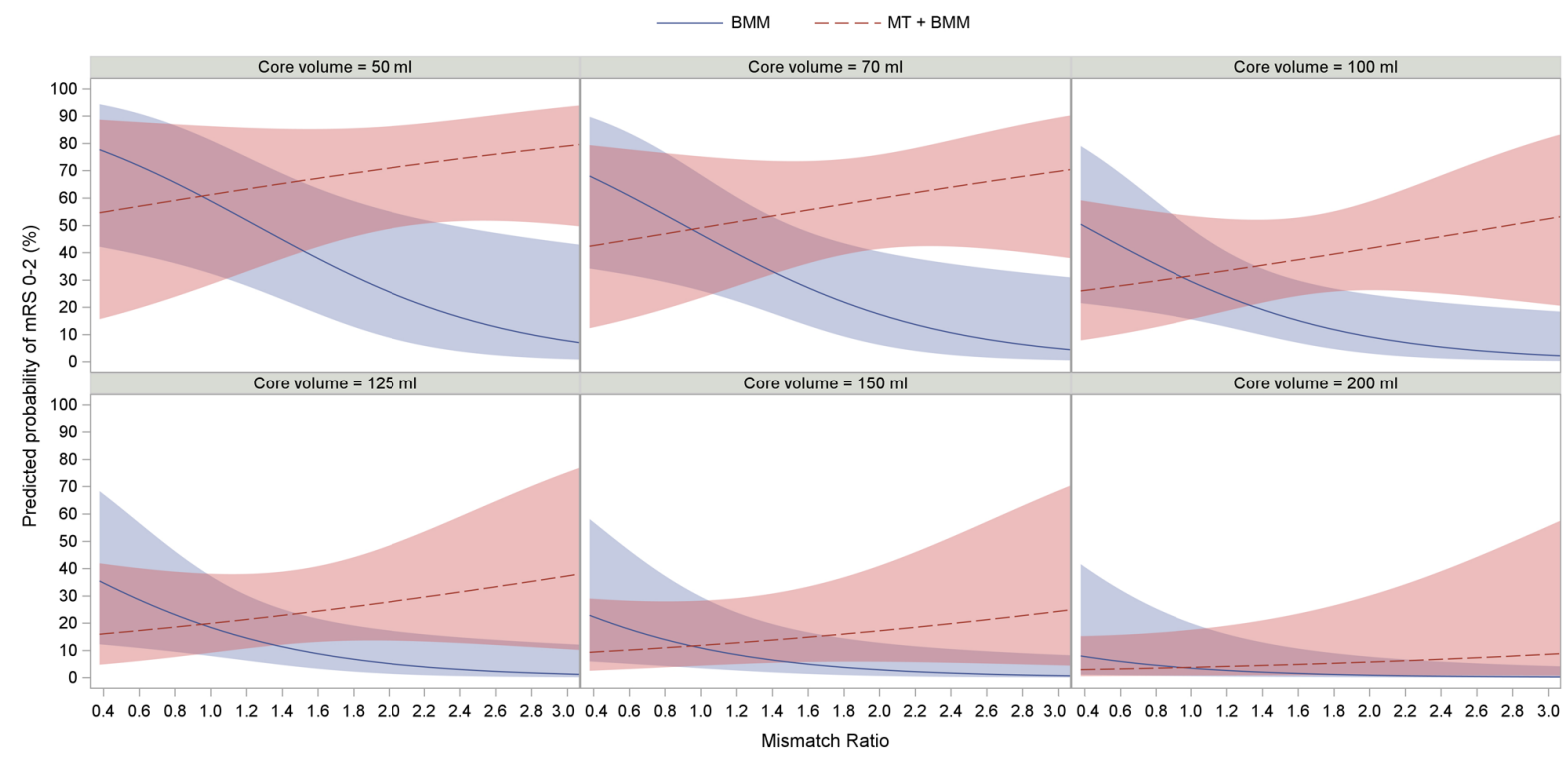

The regression curves are estimates of the probability of mRS $0-2$ according to the mismatch ratio for patients with core volume $=50 \mathrm{ml}, 70 \mathrm{ml}, 100 \mathrm{ml} 125 \mathrm{ml}, 150 \mathrm{ml}, 200 \mathrm{ml}$. The red curve corresponds to the MT+BMM group, and the blue curve to the BMM alone group. The shaded area corresponds to the 95\% confidence interval (logistic regression model).

Abbreviations: BMM indicates best medical management; MT, mechanical thrombectomy; mRS, modified Rankin scale. 
Figure 3. Predicted probability of mRS $0-2$ according to core volume and mismatch ratio in the MT+BMM group.

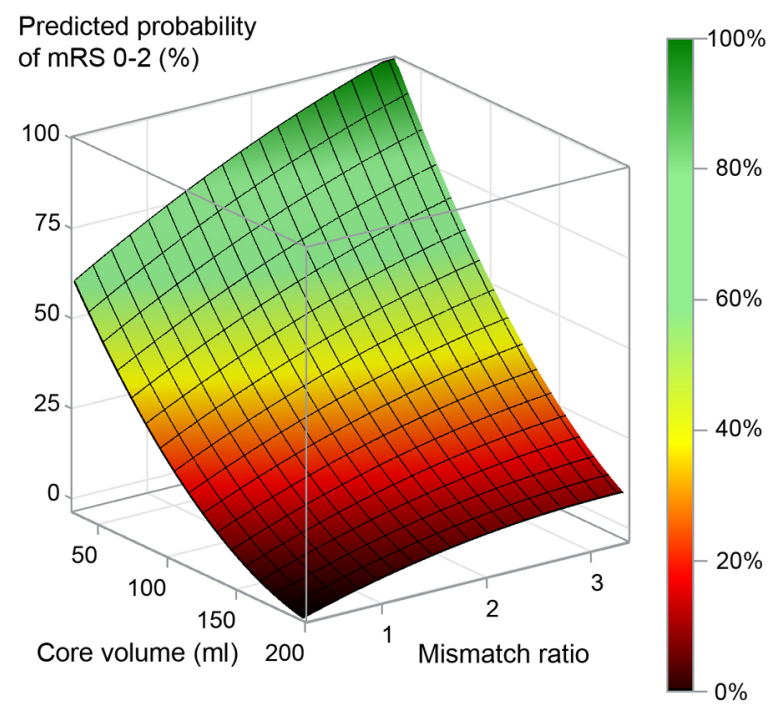


Figure 4. Ninety-days modified Rankin Scale according to treatment group and dichotomized mismatch ratio (A: 1.2 cut-off; $\mathbf{B}: 1.8$ cut-off).

A

$M T+B M M(n=23)$

MMRatio $\leq 1.2$

BMM alone $(n=23)$ 90-day modified Rankin Scale score $\square 0 \square 1 \square 2 \square 3 \square 4 \square 5 \square 6$

\begin{tabular}{|c|c|c|c|c|c|}
\hline $\begin{array}{l}4 \% \\
n=1\end{array}$ & \begin{tabular}{c|c|}
$9 \%$ & $4 \%$ \\
$n=2$ & $n=1$
\end{tabular} & $\begin{array}{l}30 \% \\
n=7\end{array}$ & $\begin{array}{l}22 \% \\
n=5\end{array}$ & $\begin{array}{l}4 \% \\
n=1\end{array}$ & $\begin{array}{l}26 \% \\
n=6\end{array}$ \\
\hline & $\begin{array}{l}26 \% \\
n=6\end{array}$ & $\begin{array}{l}22 \% \\
n=5\end{array}$ & $\begin{array}{l}17 \% \\
n=4\end{array}$ & & $\begin{array}{l}35 \% \\
n=8\end{array}$ \\
\hline
\end{tabular}

90-day modified Rankin Scale score $\square 0 \square 1 \square 2 \square 3 \square 4 \square 5 \square 6$

$M T+B M M(n=33)$

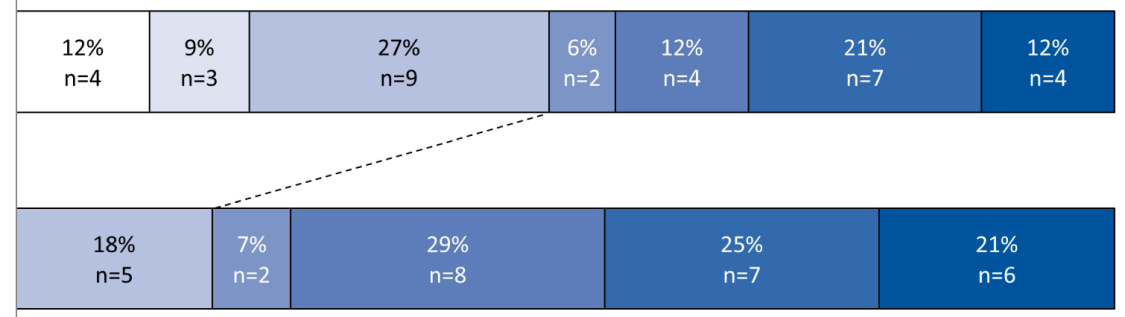

B

90-day modified Rankin Scale score

$\square 0 \square 1 \square 2 \square 3 \square 4 \square 5 \square 6$

$M T+B M M(n=36)$

\section{MMRatio <1.8}

BMM alone $(n=33)$

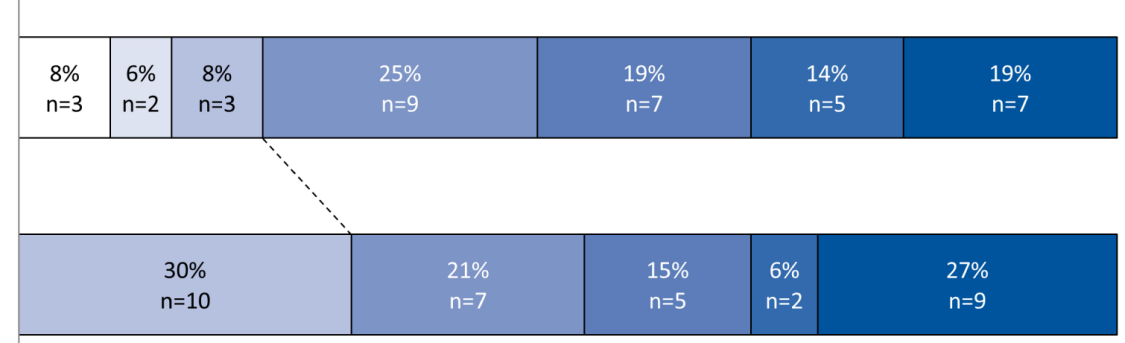

90-day modified Rankin Scale score $\square 0 \square 1 \square 2 \square 3 \square 4 \square 5 \square 6$

$M T+B M M(n=20)$

\begin{tabular}{|c|c|c|c|c|c|}
\hline $10 \%$ & $15 \%$ & $35 \%$ & $0 \% 10 \%$ & $15 \%$ & $15 \%$ \\
\hline$n=2$ & $n=3$ & $n=7$ & $=0 \quad n=2$ & $n=3$ & $n=3$ \\
\hline
\end{tabular}

MMRatio $\geq 1.8$

BMM alone $(n=18)$

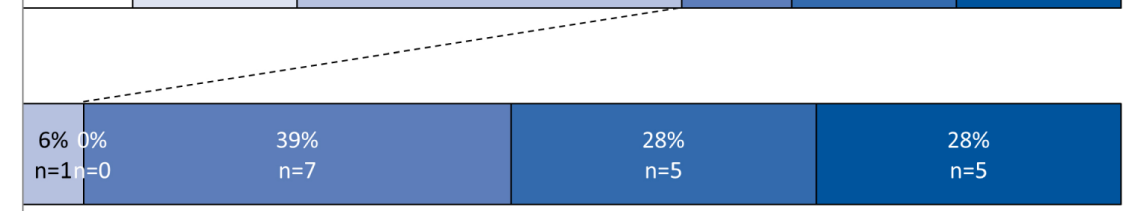

Abbreviations: MMRatio indicates mismatch ratio; BMM, best medical management; MT, mechanical thrombectomy. 


\section{References}

1. Turc G, Bhogal P, Fischer U, et al. European Stroke Organisation (ESO)- European Society for Minimally Invasive Neurological Therapy (ESMINT) guidelines on mechanical thrombectomy in acute ischemic stroke. J Neurointerv Surg. 2019 Jun;11(6):535-8.

2. Powers WJ, Rabinstein AA, Ackerson T, et al. 2018 Guidelines for the Early Management of Patients With Acute Ischemic Stroke: A Guideline for Healthcare Professionals From the American Heart Association/American Stroke Association. Stroke. 2018 Mar;49(3):e46-e110.

3. Xie Y, Oppenheim C, Guillemin F, et al. Pretreatment lesional volume impacts clinical outcome and thrombectomy efficacy. Ann Neurol. 2018 Jan;83(1):178-85.

4. Campbell BCV, Majoie C, Albers GW, et al. Penumbral imaging and functional outcome in patients with anterior circulation ischaemic stroke treated with endovascular thrombectomy versus medical therapy: a meta-analysis of individual patient-level data. Lancet Neurol. 2019 Jan;18(1):4655.

5. Rebello LC, Bouslama M, Haussen DC, et al. Endovascular Treatment for Patients With Acute Stroke Who Have a Large Ischemic Core and Large Mismatch Imaging Profile. JAMA Neurol. 2017 Jan 1;74(1):34-40.

6. Kerleroux B, Janot K, Dargazanli C, et al. Perfusion Imaging to Select Patients with Large Ischemic Core for Mechanical Thrombectomy. J Stroke. 2020 May;22(2):225-33.

7. Sarraj A, Hassan AE, Savitz S, et al. Outcomes of Endovascular Thrombectomy vs Medical Management Alone in Patients With Large Ischemic Cores: A Secondary Analysis of the Optimizing Patient's Selection for Endovascular Treatment in Acute Ischemic Stroke (SELECT) Study. JAMA Neurol. 2019 Jul 29;76(10):1147-56.

8. Kakita H, Yoshimura S, Uchida K, Sakai N, Yamagami H, Morimoto T. Impact of Endovascular Therapy in Patients With Large Ischemic Core: Subanalysis of Recovery by Endovascular Salvage for Cerebral Ultra-Acute Embolism Japan Registry 2. Stroke. 2019 Apr;50(4):901-8.

9. Mourand I, Abergel E, Mantilla D, et al. Favorable revascularization therapy in patients with ASPECTS $\leq 5$ on DWI in anterior circulation stroke. J Neurointerv Surg. 2018 Jan;10(1):5-9. 
10. Gautheron V, Xie Y, Tisserand M, et al. Outcome After Reperfusion Therapies in Patients With Large Baseline Diffusion-Weighted Imaging Stroke Lesions: A THRACE Trial (Mechanical Thrombectomy After Intravenous Alteplase Versus Alteplase Alone After Stroke) Subgroup Analysis. Stroke. 2018 Mar;49(3):750-3.

11. Olivot JM, Albucher JF, Guenego A, et al. Mismatch Profile Influences Outcome After Mechanical Thrombectomy. Stroke. 2021 Jan;52(1):232-40.

12. Saver JL, Goyal M, Bonafe A, et al. Stent-retriever thrombectomy after intravenous t-PA vs. tPA alone in stroke. N Engl J Med. 2015 Jun 11;372(24):2285-95.

13. Tisserand M, Turc G, Charron S, et al. Does Diffusion Lesion Volume Above $70 \mathrm{~mL}$ Preclude Favorable Outcome Despite Post-Thrombolysis Recanalization? Stroke. 2016 Apr;47(4):1005-11.

14. Hacke W, Kaste M, Fieschi C, et al. Randomised double-blind placebo-controlled trial of thrombolytic therapy with intravenous alteplase in acute ischaemic stroke (ECASS II). Second European-Australasian Acute Stroke Study Investigators. Lancet. 1998 Oct 17;352(9136):1245-51.

15. Campbell BC, Mitchell PJ, Kleinig TJ, et al. Endovascular therapy for ischemic stroke with perfusion-imaging selection. N Engl J Med. 2015 Mar 12;372(11):1009-18.

16. Albers GW, Marks MP, Kemp S, et al. Thrombectomy for Stroke at 6 to 16 Hours with Selection by Perfusion Imaging. N Engl J Med. 2018 Feb 22;378(8):708-18.

17. Román LS, Menon BK, Blasco J, et al. Imaging features and safety and efficacy of endovascular stroke treatment: a meta-analysis of individual patient-level data. Lancet Neurol. 2018 Oct;17(10):895-904.

18. Campbell BC, Christensen S, Parsons MW, et al. Advanced imaging improves prediction of hemorrhage after stroke thrombolysis. Ann Neurol. 2013 Apr;73(4):510-9.

19. Heit JJ, Mlynash M, Christensen S, et al. What predicts poor outcome after successful thrombectomy in late time windows? J Neurointerv Surg. 2020 May;13(5):421-425.

20. Hao Y, Liu W, Wang H, et al. Prognosis of asymptomatic intracranial hemorrhage after endovascular treatment. J Neurointerv Surg. 2019 Feb;11(2):123-6.

21. Nogueira RG, Jadhav AP, Haussen DC, et al. Thrombectomy 6 to 24 Hours after Stroke with a Mismatch between Deficit and Infarct. N Engl J Med. 2018 Jan 4;378(1):11-21. 
22. Lin L, Bivard A, Levi CR, Parsons MW. Comparison of computed tomographic and magnetic resonance perfusion measurements in acute ischemic stroke: back-to-back quantitative analysis. Stroke. 2014 Jun;45(6):1727-32.

23. d'Esterre CD, Boesen ME, Ahn SH, et al. Time-Dependent Computed Tomographic Perfusion Thresholds for Patients With Acute Ischemic Stroke. Stroke. 2015 Dec;46(12):3390-7.

24. Qiu W, Kuang H, Lee TY, et al. Confirmatory Study of Time-Dependent Computed Tomographic Perfusion Thresholds for Use in Acute Ischemic Stroke. Stroke. 2019 Nov;50(11):326973.

25. Bivard A, Kleinig T, Miteff F, et al. Ischemic core thresholds change with time to reperfusion: A case control study. Ann Neurol. 2017 Dec;82(6):995-1003.

26. Laredo C, Renú A, Tudela R, et al. The accuracy of ischemic core perfusion thresholds varies according to time to recanalization in stroke patients treated with mechanical thrombectomy: A comprehensive whole-brain computed tomography perfusion study. J Cereb Blood Flow Metab. 2020 May;40(5):966-77.

27. Austein F, Riedel C, Kerby T, et al. Comparison of Perfusion CT Software to Predict the Final Infarct Volume After Thrombectomy. Stroke. 2016 Sep;47(9):2311-7. 